\title{
Enteric Delivery of Diclofenac Sodium through Functionally Modified Poly(acrylamide-grafted-Ghatti gum)-based pH-sensi- tive Hydrogel Beads: Development, Formulation and Evaluation
}

\author{
Afrasim Moin', Talib Hussain ${ }^{2}$, DV Gowda ${ }^{3 *}$ \\ ${ }^{1}$ Department of Pharmaceutics, College of Pharmacy, University of Hail, P.O. Box 2440, Hail, KINGDOM OF SAUDI ARABIA. \\ 2Department of Pharmacology and Toxicology, College of Pharmacy, University of Hail, P.O. Box 2440, Hail, KINGDOM OF SAUDI ARABIA. \\ 3Department of Pharmaceutics, JSS College of Pharmacy, JSS University, Mysuru-570015, Karnataka, INDIA.
}

\begin{abstract}
Background: To prepare and characterize modified Poly(acrylamide-grafted-Ghatti gum). To formulate \& evaluate using this grafted polymer $\mathrm{pH}$-sensitive hydrogel beads of diclofenac sodium for enteric delivery. Methods: Polyacrylamide was grafted onto ghatti gum with microwave assistance using ceric ammonium nitrate as redox initiator. Optimization of formulation was done by varying the irradiation time, amount of initiator and acrylamide keeping amount of ghatti gum (GG) as constant. The copolymer was characterized using Fourier transform infrared spectroscopy (FT-IR), Scanning electron microscopy (SEM), Differential scanning calorimetry (DSC), elemental analysis, viscosity, molecular weight, neutralization equivalent, swelling, biodegradability and acute oral toxicity studies. Results: The copolymer was found to be biodegradable and non-toxic. Further the optimized copolymer was used to generate novel $\mathrm{pH}$ sensitive hydrogel. Diclofenac sodium (DS) was loaded into $\mathrm{pH}$ sensitive beads by ionotropic gelation technique using $\mathrm{AlCl}_{3}$ as crosslinking agent. Pulsatile swelling study indicated that the copolymer exhibits considerable $\mathrm{pH}$-sensitive behaviour
\end{abstract}

unlike pure gum. In vitro release studies were performed and it was found to be much less from copolymeric beads than from pure ghatti beads. Furthermore, a maximum of $20 \%$ drug was released from copolymeric beads in $\mathrm{pH} 1.2-5.5$ in first $3 \mathrm{~h}$ period, while $80 \%$ was released in $\mathrm{pH}$ 6.8-7.4 in $8 \mathrm{hr}$

Key words: Acrylamide grafted ghatti gum; Biodegradable; Diclofenac sodium; $\mathrm{pH}$-sensitive hydrogel; Controlled-release.

Correspondence :

Dr. D.V. Gowda, Professor, Department of Pharmaceutics, JSS College of Pharmacy, JSS University, Sri Shivarathreeshwara Nagar, Mysuru-570 015, Karnataka, INDIA

Phone: +91-9663162455

Email: dvgowda@jssuni.edu.in

DOI: 10.5530/jyp.2017.9.102

\section{INTRODUCTION}

Diclofenac sodium a non-steroidal anti-inflammatory drug (NSAID) was widely used to relieve pain, swelling, inflammation, joint stiffness/ disorders caused by arthritis and musculoskeletal disorders. It is readily absorbed from gastrointestinal tract (GIT) and has a shorter half-life of about 1.2-2 h. When administered orally, it causes certain gastric side effects such as gastric irritation, ulceration, haemorrhage, stomach pain, heart burn, nausea, vomiting, diarrhoea, indigestion, dizziness, vertigo. etc. The shorter biological half-life and associated side effects make Diclofenac sodium a suitable candidate for sustained release formulations. Oral controlled release multiple-unit dosage forms such as beads, pellets and microparticles are considered as attractive alternative to single-unit dosage forms. As they tend to spread uniformly, avoid the differences in gastric emptying and transit rates, and thereby release the drugs more uniformly throughout the GIT., ${ }^{1,2}$ On chronic dosing of a drug multiple unit dosage forms prevents the exposure of organs to higher drug concentrations unlike single unit dosage forms. ${ }^{3,4}$ They are more preferred owing to their higher degree of dispersion in GI tract, increased bioavailability, decreased dosing frequency, and reduced risk of toxicity. Moremore, local unwanted effects are eliminated when drug is embedded in a gastro-resistant polymer. This is particularly true in case of NSAIDs, which have often been associated with GIT problems. ${ }^{5,6}$

Various techniques have been reported for the preparation of multipleunit dosage forms: extrusion/- spheronization, solution or suspension layering onto sugar beads, spray-drying, liposome entrapment, coacervation, cross-linking, polymerization, single or double emulsion tech- nique, freeze drying, emulsion/solvent evaporation, hot melt extrusion and cryo pellatalization. ${ }^{7}$ Typically, microparticles in dosage forms have been prepared by emulsion/solvent evaporation technique by means of liquid paraffin, followed by rigidization and washing with large volumes of organic solvents. However, this research work avoids the use of hazardous solvents, and this process is economical, flexible and produces beads of superior quality quality. Several synthetic polymers have been used to formulate multiple-unit dosage forms. In the present decade research mainly focuses on the use and advancement of multi-unit controlled release systems using hydrophilic biopolymers, because of their abundancy and sensitivity towards modifications.

Hydrogel is a cross-linked 3D network of hydrophilic polymers which can swell without dissolving in water. The hydrogels that trigger the release of drug in response to various stimuli are known as intelligent/ smart polymers and are gaining importance in drug delivery in the present scenario as they can dictate where a drug has to be released. ${ }^{8}$ The $\mathrm{pH}$ sensitive hydrogels possess pendant acidic or basic groups that either accept or donate protons in response to changes in environmental $\mathrm{pH}$. As the $\mathrm{pH}$ of environment increases swelling of hydrogels containing acidic groups increases gradually with respect to the $\mathrm{pH}$ changes. Biopolymers are modified via grafting by monomer units. Graft copolymerization enhances the properties of natural and synthetic polymers and gives them a new property. On grafting, the host polymer attains some of the essential properties of the monomer used for grafting. The grafting of polyacrylamide (PAAM) upon the Ghatti gum is of immense importance to fabri- 
cate new materials merging the properties of both natural and synthetic polymers. Added advantage of this graft copolymer is that this could be effortlessly converted into the ionic form through the hydrolysis of amide groups resulting in $\mathrm{pH}$ sensitive copolymer. ${ }^{9}$

Gum ghatti (GG) or Indian gum is a non-starch polysaccharides, originating from India and obtained from the main species Anogeissus latifolia (Combretaceae, Myrtales), a large deciduous tree found in dry areas. ${ }^{10}$ Basing on the tests of toxicity, mutagenicity, and teratogenicity Regulatory status of GG in USA is considered as "Generally Recognized as Safe" (GRAS) since 1976. It was found to have enormously complex arrays of neutral sugar units glyceraldehyde 3-phosphate (Galp), Arabino furanose (Araf), and Arabino pyranose (Arap) and glucuronic acid (GlcA), attached to a molecular core of alternating -d-GlcA and d-Mannose residues, the former was linked through O-4 and the latter through O-2. $(1,3)$ - and $(1,6)$ - linked galactose units dwell in side chains, and the structure is dense by the presence of d-Mannose residues as double branch-points.

Although a wide range of synthetic and natural polymers have been utilised to develop oral controlled release multiple-unit dosage forms, to the best of our knowledge, there is no previous work reported on the use of functionally modified $\mathrm{pH}$ sensitive Gum Ghatti beads for Diclofenac sodium delivery to target intestine. The objective of the present research was to explore the feasibility of using functionally modified $\mathrm{pH}$-sensitive Gum Ghatti beads for targeting Diclofenac sodium to the intestine. In this study, PAAM was grafted on to the backbone of ghatti gum and consequently, the $-\mathrm{CONH}_{2}$ groups of PAAM were converted to $-\mathrm{COOH}$ groups in order to obtain a $\mathrm{pH}$-sensitive copolymer. Diclofenac sodium loaded $\mathrm{pH}$-sensitive copolymeric beads were evaluated in vitro for drug release characteristics.

\section{MATERIALS AND METHODS}

Ghatti Gum (GG) was purchased from Sigma Aldrich, Mumbai, India. Ceric ammonium nitrate (CAN) and Polyacrylamide (PAAM) were obtained from Merck Specialties Pvt. Ltd, Mumbai, India. Diclofenac Sodium was obtained as gift sample from Sigma Aldrich, Mumbai. Acetone (density $=0.789-0.791 \mathrm{~g} / \mathrm{mL}$ ) was bought from Loba Chemie, Mumbai, India. All other chemicals and reagents critical for the synthesis and evaluations were procured from Merck Specialties Pvt. Ltd (Mumbai, India), unless otherwise specified. Throughout the experiment, Millipore water was used.

\section{Preparation of polyacrylamide grafted GG}

The graft copolymer obtained from PAAM and GG was prepared by free radical induced, microwave-assisted copolymerization method. ${ }^{11}$ Precise amount of polyacrylamide (Table 1) was mixed with $30 \mathrm{ml}$ of water and added to aqueous dispersion of GG $(0.0079 \mathrm{~g} / \mathrm{ml})$ and stirred for about 1 h. A specified amount of CAN (Table 1) was dissolved in $30 \mathrm{ml}$ of water and mixed in the previous dispersion of gum and monomer. The dispersion was irradiated by means of microwave (laboratory scientific microwave system, Catalyst systems, India) at $480 \mathrm{~W}$ for different time periods using alternate one minute heating and one minute cooling cycles. The irradiated samples were then left all night at ambient temperature and then precipitated using acetone. The precipitate was further washed with ethanol to ensure that the precipitate is devoid of unreacted acrylamide monomer and homopolymer. The prepared copolymer was vacuum dried at $40^{\circ} \mathrm{C}$ until a constant weight is attained and then converted to fines. The Grafting Efficiency (GE) and Percentage Grafting were calculated using following equations:

$$
\begin{gathered}
\mathrm{GE}(\%)=\frac{w 1-w 0}{w 2} \times 100 \\
\% \mathrm{G}=\frac{w 1-w 0}{w 0} \times 100
\end{gathered}
$$

Where, $\mathrm{W}_{0}, \mathrm{~W}_{1}$ and $\mathrm{W}_{2}$ denote the weight of GG, copolymer and PAAM, respectively. Various reaction parameters were optimized as a function of percentage grafting. Reaction time, temperature and amount of initiator were optimized with respect to percentage grafting $(\mathrm{Pg})$, whereas concentration of PAAM was optimized with respect to percentage swelling (Ps).

\section{Full factorial design}

Factorial design is a broadly used experimental design comprising of different levels and factors. ${ }^{12}$ Different levels of a given factor are combined with all levels of every other factor in the experiment. In the present study, two-level, three-factor, full factorial design (comprising of 8 batches) was used for the optimization of polyacrylamide grafting onto GG. The amount of polyacrylamide, ceric ammonium nitrate and microwave exposure time were selected as independent variables and the percentage of nitrogen $(\% \mathrm{~N})$ in the grafted gum, its viscosity and swelling behaviour were selected as responses.

The $\% \mathrm{~N}$ was expected to ascend in copolymer due to the presence of high nitrogen content in polyacrylamide which is grafted onto the pure GG. The grafting of amide onto GG was envisaged by changes in viscosity, swelling behaviour and hydrophilicity of the pure gum. Each independent variable was examined at two levels, high level $(+1)$ and low level $(-1)$. Polynomial models were produced for all the response variables through Multiple Linear Regression Analysis (MLRA) approach. The results of responses generated using the experimental designs were

Table 1: Synthesis details of acrylamide grafted GG

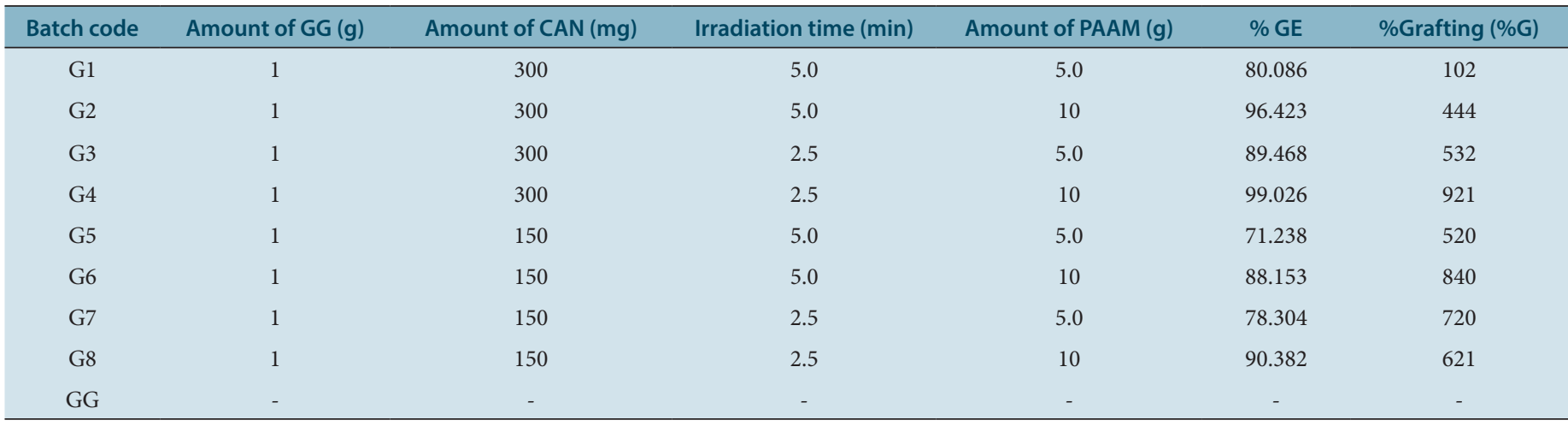


analyzed by factorial models using Design Expert software (Version 7.0.0, Stat-Ease, Inc., Minneapolis).

\section{Alkaline hydrolysis of GG-g-PAAM copolymer}

Optimised GG-g-PAAM copolymer ( $2 \mathrm{~g})$ was dissolved in a precise volume of $\mathrm{NaOH}$ solution $(0.9 \mathrm{M})$ and stirred at $75^{\circ} \mathrm{C}$ for $60 \mathrm{~min}$ in a water bath. At the completion of reaction, the solution was cooled and poured in excess methanol. The hydrolyzed copolymer was separated by filtration, washed thoroughly with methanol and dried overnight at $50{ }^{\circ} \mathrm{C}^{13}$ The feasible reaction scheme is shown in Figure 1.

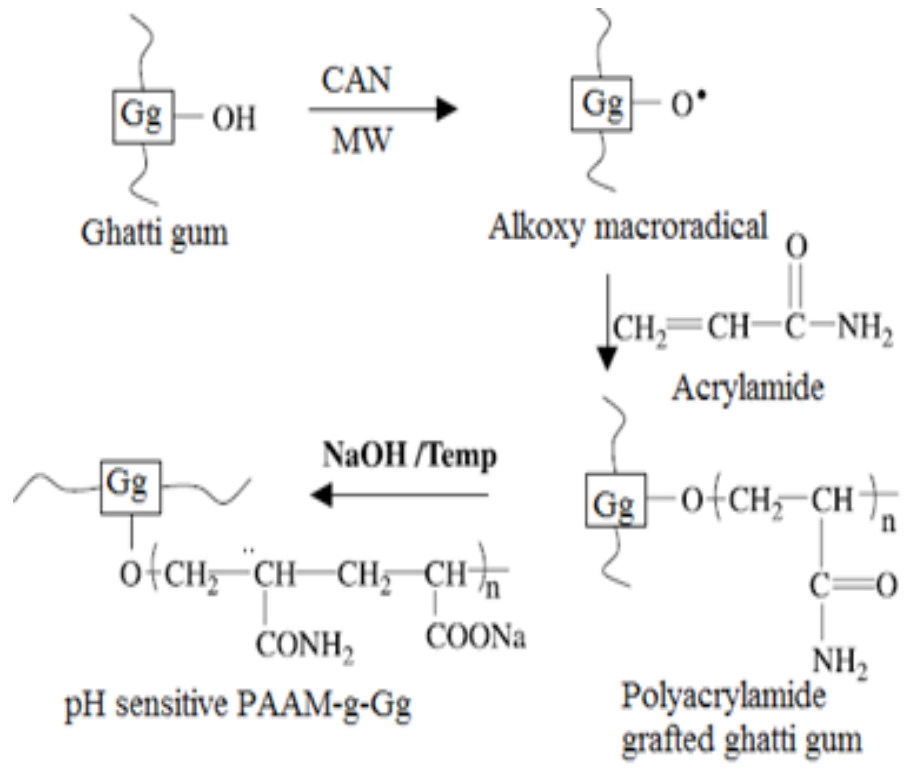

Figure 1: Proposed mechanistic pathway for the synthesis of $\mathrm{pH}$-sensitive PAAM-g-GG copolymer.

\section{Characterization of the copolymer}

\section{Fourier transform infrared spectroscopy (FTIR)}

FTIR was performed to characterize the presence of specific functional groups in the material. $0.5^{-1} \mathrm{~mm}$ thick films of GG and all copolymers (G1-G8) were prepared and analyzed by FTIR using transmittance mode. ${ }^{14}$ Infrared spectra were obtained in the range of wave numbers from 4000 to $650 \mathrm{~cm}^{-1}$ all through 64 scans, under $2 \mathrm{~cm}^{-1}$ resolution (FTIR-8400S, Shimadzu, Japan).

\section{Elemental analysis}

The elemental analysis of GG, all the copolymers (GG-g-PAAM 1 to GG-g-PAAM 8) and hydrolysed grafted gum was performed by using a PCHN 2400 micro-analyser (Perkin Elmer, USA). The carbon, hydrogen and nitrogen contents were calculated.

\section{Differential scanning calorimetry (DSC)}

DSC thermograms of GG and different batches of GG-g-PAAM were obtained using DSC-60 (Shimadzu, Japan) by heating the samples from $10^{\circ} \mathrm{C}$ to $300^{\circ} \mathrm{C}$ under nitrogen purge $(50 \mathrm{ml} / \mathrm{min})$ at a heating rate of $10^{\circ} \mathrm{C} / \mathrm{min}$.

\section{Scanning electron microscopy (SEM)}

The morphology of the GG, GG-g-PAAM and P5 beads was examined using SEM system (LEO 435 VP, CFTRI, Mysuru). The samples were gold coated to enhancethe conductivity of the electron beam. An accelerating voltage of $10 \mathrm{kV}$ and a working distance of $12 \mathrm{~mm}$ were used. The coated beads were observed under scanning electron microscope (SEM) (LEO $435 \mathrm{VP}$, CFTRI, Mysuru) at the requisite magnification at room temperature.

\section{Intrinsic viscosity measurement}

Viscosity of the polymer solutions was measured using programmable Brookfield viscometer (Model: Brookfield CAP-2000+ viscometer, Brookfield Engineering Labs, Inc., USA) at $32.7^{\circ} \mathrm{C}$ and at neutral $\mathrm{pH} .{ }^{15}$ The time of flow for solutions was measured at four different concentrations $(0.1 \%, 0.05 \%, 0.025 \% \& 0.0125 \%)$. From the time of flow of polymer solutions $(t)$ and that of the solvent $\left(t_{0}\right.$, for distilled water), relative viscosity $\left(\eta_{\text {rel }}=t / t_{0}\right)$ was obtained. Specific viscosity $\left(\eta_{\text {sp }}\right)$, reduced viscosity $\left(\eta_{\text {red }}\right)$ and inherent viscosity $\left(\eta_{\text {inh }}\right)$ were mathematically calculated as shown in equation 3,4 and 5 .

$$
\begin{gathered}
\eta_{\text {sp }}=\eta_{\text {rel }}-1 \\
\eta_{\text {red }}=\frac{\eta \text { sp }}{c} \\
\eta_{\text {inh }}=\frac{\ln \eta \text { rel }}{C}
\end{gathered}
$$

Where ' $C$ ' represents polymer concentration in $\mathrm{g} / \mathrm{dL}$.

The reduced viscosity $\left(\eta_{\text {red }}\right)$ and the inherent viscosity $\left(\eta_{\text {inh }}\right)$ were simultaneously plotted against concentration. The intrinsic viscosity was calculated from the point of intersection after extrapolation of two plots (i.e. $\eta_{\text {red }}$ versus $C$ and $\eta_{\text {inh }}$ versus C) to zero concentration.

\section{Molecular weight analysis}

The molecular weight of sample was found out using gel permeation chromatographic technique (1100 series, Agila Technologies, UK) via refractive index detector. A PL aquagel- $\mathrm{OH}$ mixed column $(7.5 \mathrm{~mm} \times 300$ mm; $8 \mu \mathrm{m}$; Agila Technologies, United Kingdom) was used with mobile phase consisting of $0.1 \%$ w/w Sodium azide (Ajax Fine chem, Australia) dissolved in de-ionized water. The flow rate of mobile phase and the column temperature were maintained constantly at kept at $0.5 \mathrm{ml} / \mathrm{min}$ and $30{ }^{\circ} \mathrm{C}$ respectively. Dextrans (Sigma Aldrich, Germany) with molecular weights of 150,000, 410,000, 670,000, 1,400,000 and 2,000,000 Da were used as standards. Prior to the analysis $1 \mathrm{mg} / \mathrm{ml}$ of standard as well sample solutions were filtered through a cellulose nitrate membrane (pore diameter $=0.45 \mu \mathrm{m}$ ). Average results of triplicate samples were reported.

\section{Swelling studies in deionized water}

Various parameters such as irradiation time, temperature and $\mathrm{pH}$ were optimised through swelling studies of GG-g-PAAM at varied time intervals $(4,8,12,16,20$ and $24 \mathrm{~h})$, varied temperatures (30, 40, 50, 60 and $\left.70^{\circ} \mathrm{C}\right)$ and $\mathrm{pH}(3.0-13.0)$. Initially required amount of sample (100 mg) was dried in a hot air oven for $24 \mathrm{~h}$ at $40^{\circ} \mathrm{C}$, weighed, and then it was immersed into $100 \mathrm{ml}$ of deionised water. ${ }^{16}$

After a predetermined time interval the sample was taken out, wiped gently and reweighed. Ps was calculated as per equation 6 . Following the optimization of time, effect of temperature on Ps was studied at pre-optimized time subsequently the optimization of $\mathrm{pH}$ at pre-optimized time and temperature was determined.

$$
\mathrm{Ps}_{\mathrm{s}}=\frac{\mathrm{w}_{\mathrm{s}}-\mathrm{w}_{\mathrm{d}}}{\mathrm{w}_{\mathrm{d}}} \times 100
$$


Where $\mathrm{Ws}$ and $\mathrm{W}_{\mathrm{d}}$ are the weights of the swelled and dry polymers, respectively and Ps is percentage swelling. The experiment was carried out in triplicates.

\section{Swelling studies in different salt solutions}

Effect of ionic strength on the swelling behaviour of the copolymer was studied at pre-optimised time, temperature and $\mathrm{pH}$ using different salt solutions of $\mathrm{NaCl}, \mathrm{BaCl}_{2}, \mathrm{FeCl}_{3}$ and $\mathrm{SnCl}_{4}$ in deionised water having 0.01 , $0.02,0.03,0.04$ and $0.05 \mathrm{~mol} \mathrm{~L}^{-1}$ ionic strength of cations i.e. $\mathrm{Na}^{+}, \mathrm{Ba}^{2+}$ $\mathrm{Fe}^{3+}$ and $\mathrm{Sn}^{4+}$. Measured quantity of dry sample was immersed in salt solutions and kept under pre-optimized swelling conditions. Finally the samples were taken out wiped carefully and re-weighed. Ps was calculated as per equation 6 .

\section{Neutralisation equivalent}

Titration method was widely used to estimate the NE (Tripathy and Singh 2000). Two hundred milligrams of the samples were equilibrated with $0.1 \mathrm{~N} \mathrm{HCl}$ for $6 \mathrm{~h}$ and the surplus hydrogen ion concentration was back titrated with a standard Sodium hydroxide solution $(0.1 \mathrm{~N})$. Subsequently, the equivalent weight of the carboxylic acid functional group was calculated. ${ }^{17}$

\section{Preparation of beads}

DS equivalent to 20 or $40 \%$ of dry mass of the polymer was precisely measured and dispersed in 3\% w/v aqueous solution of GG or optimised copolymer and mixed thoroughly by means of magnetic stirrer. Using $25 \mathrm{ml}$ hypodermic syringe with needle 23 , hydrolysed copolymer dispersion and GG solution were poured into $100 \mathrm{ml}$ aqueous solutions of $\mathrm{AlCl}_{3}(20,30$ and $40 \% \mathrm{w} / \mathrm{v})$ at a rate of $25 \mathrm{drops} / \mathrm{min}$. The dispersion was continuously stirred and the beads formed were separated from gelation medium, washed twice with distilled water to get rid of unreacted ions and dried at room temperature for $24 \mathrm{~h}$ and then at $40{ }^{\circ} \mathrm{C}$ for $10 \mathrm{~h}$. The composition of beads is given in Table 2. Drug-free co-polymeric beads were prepared following the same method.

Table 2: Composition of beads

\begin{tabular}{ccccc}
\hline Code & \multicolumn{2}{c}{ Polymer (\%w/v) } & $\begin{array}{c}\text { Drug }(\% \text { w/w of } \\
\text { polymer })\end{array}$ & AICl3 (\% w/v) \\
\cline { 2 - 5 } & GG & GG-g-PAAM & & \\
\hline P1 & 3 & - & 20 & 20 \\
P2 & - & 3 & 20 & 20 \\
P3 & - & 3 & 20 & 30 \\
P4 & - & 3 & 20 & 40 \\
P5 & - & 3 & 40 & 40 \\
\hline
\end{tabular}

\section{Measurement of bead size}

Digimatic Micrometer (MDC-25S Mitutoyo, Japan) with an accuracy of $0.001 \mathrm{~mm}$ was used to determine the bead size. The average diameter of 100 particles per batch was calculated.

\section{Estimation of drug entrapment efficiency}

A measured quantity of beads were incubated in $100 \mathrm{ml}$ of $\mathrm{pH} 7.4$ phosphate buffer solution for complete swelling and sonicated via probe sonicator (Toshiba, DP120, New Delhi, India) for 15 min to rupture the beads and facilitate the extraction of the drug. The solution was heated gently for $3 \mathrm{~h}$, cooled and centrifuged using a centrifuge (C24 BL-Remi, India). The supernatant liquid was separated; the residue was washed twice with fresh buffer solution to remove any residual drug and added to the supernatant liquid. The pooled liquid was estimated for the drug content using UV-visible spectrophotometer (Model UV-1800, Shimad$\mathrm{zu}$, Japan) at $260 \mathrm{~nm}$.

\section{Pulsatile swelling study}

The pulsatile swelling behaviour of drug-free copolymer beads and GG beads were studied by measuring their mass in $25 \mathrm{ml}$ solutions of $\mathrm{pH} 1.2$ and 7.4 at $37^{\circ} \mathrm{C}$. A random sequence of step changes was used to analyze the mass response. The $\mathrm{pH}$ of the surrounding medium was changed alternatively. The beads were taken out at predetermined time intervals, and excess surface liquid was wiped cautiously using tissue paper and weighed using an electronic balance (Model BL 220H, Shimadzu, Japan) having an accuracy of $0.001 \mathrm{mg}$. The percentage of water uptake (Q) at different time intervals was calculated using the equation 7 .

$$
\mathrm{Q}=\frac{\mathrm{W}_{2}-\mathrm{W}_{1}}{\mathrm{~W}_{1}} \times 100
$$

Where $\mathrm{W}_{1}$ is the mass of the dry beads and $\mathrm{W}_{2}$ is the mass of the swollen beads.

\section{In vitro drug release studies}

One set of dissolution study was carried out with weighed quantities of beads in $900 \mathrm{ml}$ of dissolution medium $(0.1 \mathrm{~N} \mathrm{HCl}$ solution of $\mathrm{pH} 1.2$ and $\mathrm{pH} 7.4$ phosphate buffer solution) at $37 \pm 0.5^{\circ} \mathrm{C}$ using a programmable dissolution tester (paddle type, ElectrolabTDT-06P (USP) Mumbai, India) at $50 \mathrm{rpm}$. Second set of dissolution test was performed to evaluate the effect of constant $\mathrm{pH}$ changes with time (i.e. to simulate gastrointestinal $\mathrm{pH}$ changes), at $37 \pm 0.5^{\circ} \mathrm{C}$ in $900 \mathrm{ml}$ of dissolution medium of different $\mathrm{pH}$ values ( $\mathrm{pH} 1.2$ for $2 \mathrm{~h}, \mathrm{pH} 5.5$ for $1 \mathrm{~h}, \mathrm{pH} 6.8$ for $2 \mathrm{~h}$, followed by $\mathrm{pH} 7.4$ for $3 \mathrm{~h}$ ) Aliquots of samples were removed at predefined time intervals and were replenished immediately with fresh media to maintain sink conditions. The aliquots, following filtration through a $0.45 \mathrm{~mm}$ membrane filter and after suitable dilution, were analyzed at $260 \mathrm{~nm}$ using UV-visible spectrophotometer.

\section{Biodegradability study}

$5 \%$ gum solution was poured onto petri dish and dried. Samples were cut $(2 \mathrm{~cm} \times 2 \mathrm{~cm})$ and placed on the agar medium devoid of any carbon source. Aspergillus niger was inoculated on to the sample films of GG-gPAAM and incubated at ambient temperature $\left(37^{\circ} \mathrm{C}\right)$ for 21 days. Thereafter, the films were observed for evidence of colony growth.

\section{Acute oral toxicity study}

Acute oral toxicity study of GG-g-PAAM was performed as per the "Organization of Economic Co-operation and Development (OECD) guidelines for the test of chemicals" 425, adopted "17 December 2001". The study protocol was prior approved by the Institutional Animal Ethics Committee (IAEC) of JSS College of Pharmacy, JSS University, Mysuru, India. Five nulliparous and non-pregnant five weeks old female albino rats (Swiss strain) were taken for this study. Rats were housed in polycarbonate cage with food and deionised reverse osmosis water at $20-25^{\circ} \mathrm{C}$ and $40-70 \%$ relative humidity in a $12 \mathrm{~h} \mathrm{light/dark} \mathrm{cycle.} \mathrm{A} \mathrm{single} \mathrm{dose} \mathrm{of}$ $2000 \mathrm{mg} / \mathrm{kg}$ body weight of GG-g-PAAM was administered by gavages using a stomach tube to the first animal. If the first animal survives then the same dose was administered to the remaining four animals. The animals were observed at specified time intervals upto 14 days. The mortality rate was assessed by visible observation and reported accordingly. 


\section{RESULTS AND DISCUSSION}

\section{Synthesis of GG-g-PAAM by microwave assisted method}

Synthesis of polyacrylamide grafted GG was carried out via graft copolymerization. Ceric ammonium nitrate is a commonly used reagent to initiate free radical graft copolymerization. As CAN is an electron deficient molecule it acquires electrons from alcoholic oxygen in GG to form a new bond i.e. Ce-O (GG-ceric complex). The ceric (IV) ions in the complex get reduced to ceric (III) ions by oxidizing hydrogen atom. As it is a highly polar bond (than O-H bond), it has affinity to breakdown easily on microwave irradiation and forms free radical on the backbone of GG, and the grafting starts right from the free radical end. The anticipated mechanism of microwave assisted grafting has been shown in Figure 2.

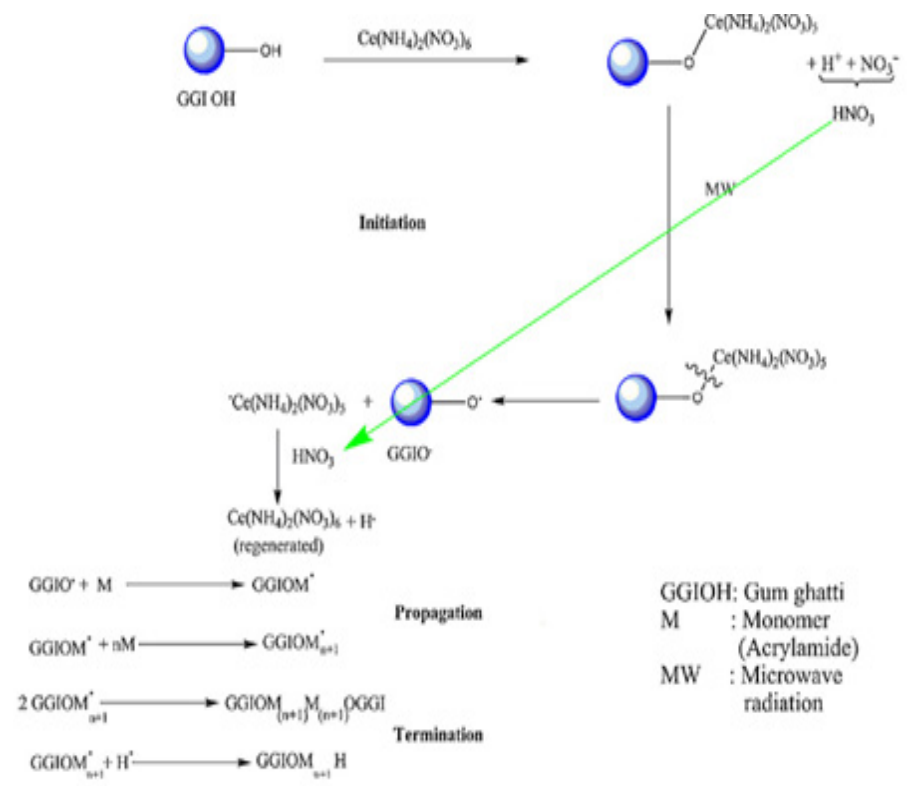

Figure 2: Schematic representation of microwave assisted synthesis of GGg-PAAM.

A considerable amount of redox initiator is essential for free radical generation. Free radical readily reacts with the monomer and plays a prominent role in grafting of PAAM onto GG. GG free radical chemically couples to the monomer unit via covalent bond. The reaction is terminated by combination of two radicals. The sample is then provided with microwave irradiation which greatly reduces the reaction time by inducing rapid internal energy transfer. Microwave is regarded as a catalyst which synergises with ceric ions in graft copolymerization.

The optimized formulation has been determined by its higher percentage grafting and intrinsic viscosity (which is directly proportional to the molecular weight). Using factorial design three different variables were picked out to optimize the GE. MLRA was used to express the mathematical relationship with GE.

$$
\mathrm{GE}(\%)=76.92+5.27 \mathrm{~A}-2.54 \mathrm{~B}+6.58 \mathrm{C}
$$

Where, A is amount of CAN (mg), B is irradiation time (min) and C is amount of PAAM $(\mathrm{g})$. The positive effect of CAN and PAAM was due to the formation of more number of free radical sites and addition of PAAM side chains to them. The negative effect of irradiation time on GE was owing to chain breakage under microwave irradiation. ANOVA analysis showed that the factorial model was significant $(\mathrm{P}<0.04)$ having $\mathrm{R}^{2}$ value of 0.97 . The adjusted (0.98) and the predicted $(0.96) \mathrm{R}^{2}$ values were in realistically good agreement.

\section{Characterization of the copolymer}

\section{Optimization of various reaction parameters}

Effects of various reaction parameters such as reaction time, temperature, initiator concentration, concentration of PAAM on the graft copolymerization were accounted in Figure 3. Figure 3(a) illustrates the effect of reaction time on Pg and the reaction time was varied from 60 to $300 \mathrm{sec}$. It was observed that percentage grafting increases with increase in reaction time reaches a maximum point (79\%) after $150 \mathrm{sec}$ and then decreases with further increase in reaction time. This can be inferred as primarily with the increase in reaction time, interactions between ${ }^{\star} \mathrm{OH}$ and substrate increased leading to the formation of more active sites on GG as well as on PAAM chains and the rate of grafting was very high which resulted in increased Pg. Whereas, at too longer reaction time, concentration of PAAM and radicals reduced, further homo-polymerization pre-dominates over graft copolymerization. Moreover, homopolymerization results in highly viscous medium and forms a barrier in the path of the free radicals reaching the active sites. Hence, a decreased Pg was observed after optimal reaction time. ${ }^{18}$
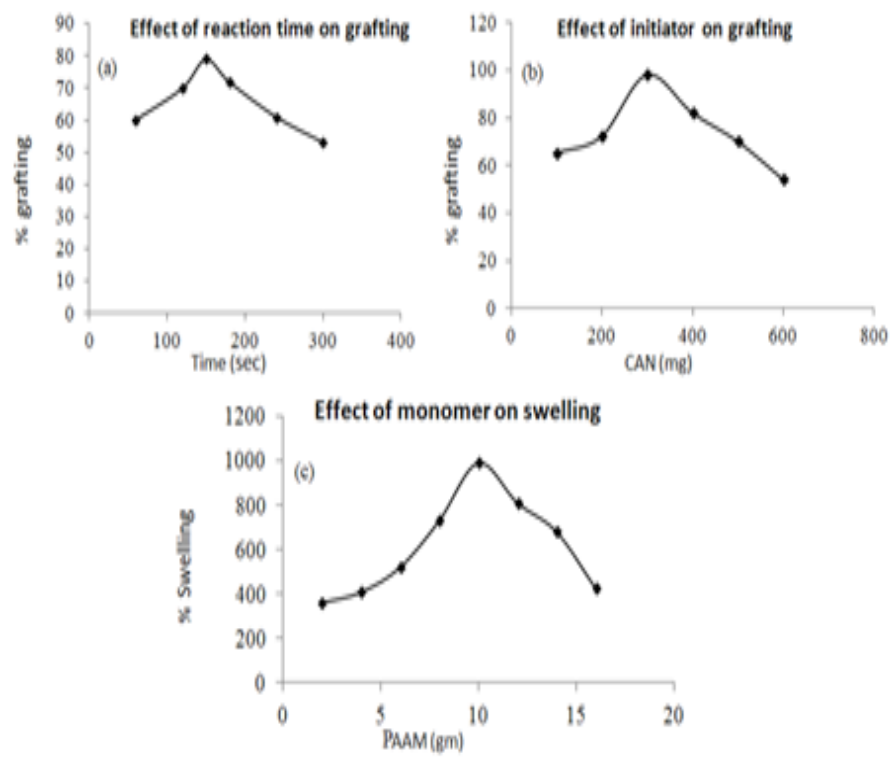

Figure 3: (a) Effect of reaction time on Pg (b) effect of initiator concentration on Pg (c) effect of concentration of PAAM on Ps.

Concentration of initiator was also plays a prominent role in depicting the grafting yield. Figure 3(b) demonstrates the effect of initiator concentration on Pg. Maximum Pg was attained at $300 \mathrm{mg}$ of CAN. However, further increase resulted in decreased grafting yield. Initially concentration of initiator was augmented leading to the production of more active sites on the PAAM chains and GG resulting in increased Pg, whereas, excessive concentration of the initiator led to the premature cessation of the reaction and did not allowed further grafting. Therefore, lesser Pg was observed at higher concentration of initiator.

Optimization of the concentration of PAAM was necessary to obtain a hydrogel with maximum swelling capacity and was done as a function of Ps. Figure 3(c) discloses the effect of PAAM concentration on Ps. Concentration of PAAM was varied from 0.028 to $0.225 \mathrm{moll}^{-1}$ (2-16 
gm) by keeping remaining grafting parameters constant. Initially Ps was increased with increase in PAAM concentration and attained maximum value at $0.140 \mathrm{moll}^{-1}(10 \mathrm{gm})$ concentration of PAAM whereas, a sharp decline in the Ps was observed with the further increase in PAAM concentration. The initial increase in swelling may be attributed to the highly porous and flexible network structure that could hold more water molecules however further increase in PAAM concentration resulted in firm and dense crosslinked moiety, thereby showing lesser swelling. ${ }^{19}$

\section{FTIR spectroscopy}

From the FTIR spectrum of gum ghatti [Figure 4(a)], it has been observed that a peak at $3411.69 \mathrm{~cm}-1$ is due to stretching vibration of $2^{0}$ $-\mathrm{OH}\left(\mathrm{CH}_{2} \mathrm{OH}\right)$, Smaller peaks at $2928.04 \mathrm{~cm}^{-1}$ are assigned to the $\mathrm{C}-\mathrm{H}$ stretching vibrations. Peak at 1855.58 and $1618.33 \mathrm{~cm}^{-1}$ are assigned to $-\mathrm{C} \equiv \mathrm{C}-, \mathrm{C}-\mathrm{O}$ stretching vibrations. The peak at $1421.58 \mathrm{~cm}^{-1}$ is due to $-\mathrm{COO}$ - bending vibration, $1032.62 \mathrm{~cm}^{-1}$ is attributed to the $\mathrm{C}-\mathrm{O}-\mathrm{C}$ stretching vibration, 777.34 peak is due to pyranose ring structure.

In case of GG-g-PAAM (G4) [Figure 4(b)], it has been observed that there are some additional peaks. Peak at $3450.82 \mathrm{~cm}^{-1}$ indicates N-H group of amide, 2929.97 is due to $\mathrm{C}-\mathrm{H}$ stretching vibrations; band at $1658.84 \mathrm{~cm}^{-1}$ is attributed to amide-I (CO stretching). The peak at $1350.22 \mathrm{~cm}^{-1}$ is due to $\mathrm{C} \mathrm{N}$ stretching vibrations. The peak appearing at $1122.61 \mathrm{~cm}^{-1}$ is due to the ether linkage formed by the reaction between $\mathrm{OH}$ groups of GG and PAAM is a supportive evidence for grafting. This confirms the grafting reaction. FTIR analysis indicated that GG was successfully modified into GG-g-PAAM.

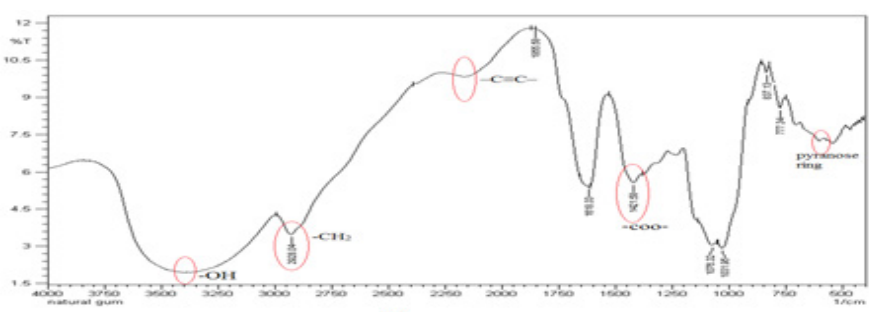

(a)

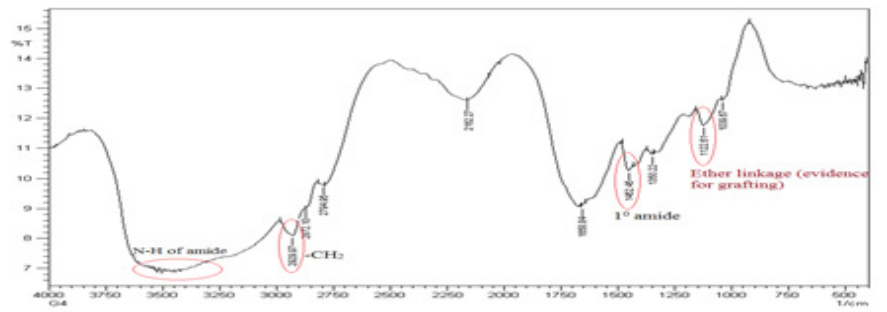

(b)

Figure 4: FTIR spectrum of (a) pure GG and (b) optimized GG-g-PAAM (G4).

\section{Elemental analysis}

The results of elemental analysis (C, H \& N) for GG and of all batches of polyacrylamide grafted ghatti gum (GG-g-PAAM) are shown in Table 3. Gum ghatti by nature is devoid of nitrogen. However, all the batches of GG-g-PAAM have nitrogen. The presence of nitrogen in GG-g-PAAM (but absent in GG) is an indication of grafting of PAAM chains on to GG as the amide groups present in PAAM chains contribute to the nitrogen in GG-g-PAAM). Further, as percentage grafting increases, percentage of nitrogen increases. A considerable increase in nitrogen (16.75\%) was seen in G4. This can be attributed to the presence of $-\mathrm{CONH}_{2}$ groups of PAAM on the GG. On the other hand, the percentage of nitrogen was reduced to $7.26 \%$ in hydrolyzed copolymer. During hydrolysis, the $-\mathrm{CONH}_{2}$ groups were converted to - $\mathrm{COOH}$ groups, leading to decreased nitrogen content. This confirmed the grafting and hydrolysis reactions.

\begin{tabular}{cccc}
\multicolumn{4}{c}{ Table 3: Elemental analysis details of PAAM-g-GG and native GG } \\
\hline Batch code & \%C & \%H & $\% \mathbf{N}$ \\
\hline G1 & 42.31 & 8.71 & 9.45 \\
G2 & 43.62 & 9.13 & 12.25 \\
G3 & 44.51 & 9.36 & 12.82 \\
G4 & 46.89 & 10.82 & 16.75 \\
G5 & 44.38 & 9.69 & 11.97 \\
G6 & 43.87 & 9.42 & 15.84 \\
G7 & 42.75 & 9.63 & 13.75 \\
G8 & 42.27 & 10.12 & 13.08 \\
GG & 38.42 & 8.45 & 0.00 \\
Hydrolysed gum & 46.52 & 14.37 & 7.26 \\
\hline
\end{tabular}

Mathematical relationship generated using MLRA for $\% \mathrm{~N}$ was expressed as (Equation 9).

$$
\% \mathrm{~N}=15.95+0.92 \mathrm{C}
$$

Where $\mathrm{C}$ is the amount of PAAM (g).

Since nitrogen was present only in PAAM amount of CAN and irradiation time becomes insignificant in estimation of \% $\mathrm{N}$ content. An increase in \% $\mathrm{N}$ with \% GE indicates the grafting of PAAM onto the GG backbone. From ANOVA study, it was observed that the factorial model was significant $(\mathrm{P}<0.05)$ having $\mathrm{R}^{2}$ value of 0.86 . The adjusted $(0.82)$ and the predicted $(0.89) \mathrm{R}^{2}$ values were in convincingly good agreement.

\section{Thermogravimetric analysis, Differential thermal analysis, Differential thermogravimetric analysis (TGA/ DTA/DTG)}

TGA of GG and GG-g-PAAM was studied as a function of \% weight loss versus temperature. Decomposition was observed in case of TGA of GG and GG-g-PAAM. GG showed an initial decomposition temperature (IDT) of $206.9^{\circ} \mathrm{C}$ and final decomposition temperature (FDT) at $521.6^{\circ} \mathrm{C}$ [Figure 5(a) $]^{20}$, whereas; GG-g-PAAM showed IDT and FDT at $134.7^{\circ} \mathrm{C}$ and $598.7^{\circ} \mathrm{C}$ respectively [Figure $5(\mathrm{~b})$ ].

From the comparison of the TGA of GG and GG-g-PAAM, it was observed that the IDT of GG was elevated whereas the FDT was lower than that of GG-g-PAAM. This may be due to the grafting of PAAM which lowers the IDT of the graft copolymers, and the PAAM chains degrade by the formation of imide group and evolution of ammonia. Formation of imide group increases the FDTs of the graft copolymers. Thus, it could be concluded from the TGA data that GG-g-PAAM is thermally more stable as compared to GG because of the formation of crosslinks between different polymeric chains through covalent bonding. The results furnished by the TGA studies were further supported by DTA and DTG studies.

The exothermic peak at $483.2^{\circ} \mathrm{C}(203 \mu \mathrm{V})$ was obtained in case of DTA of GG, whereas; GG-g-PAAM exhibited exothermic DTA peaks at 597.3 ${ }^{\circ} \mathrm{C}(186.6 \mu \mathrm{V})$. From the DTA data, it is clear that the heat evolved in the thermal decomposition of GG-g-PAAM is very less as compared to GG. Moreover, in case of GG-g-PAAM degradation occurred at higher temperature. 


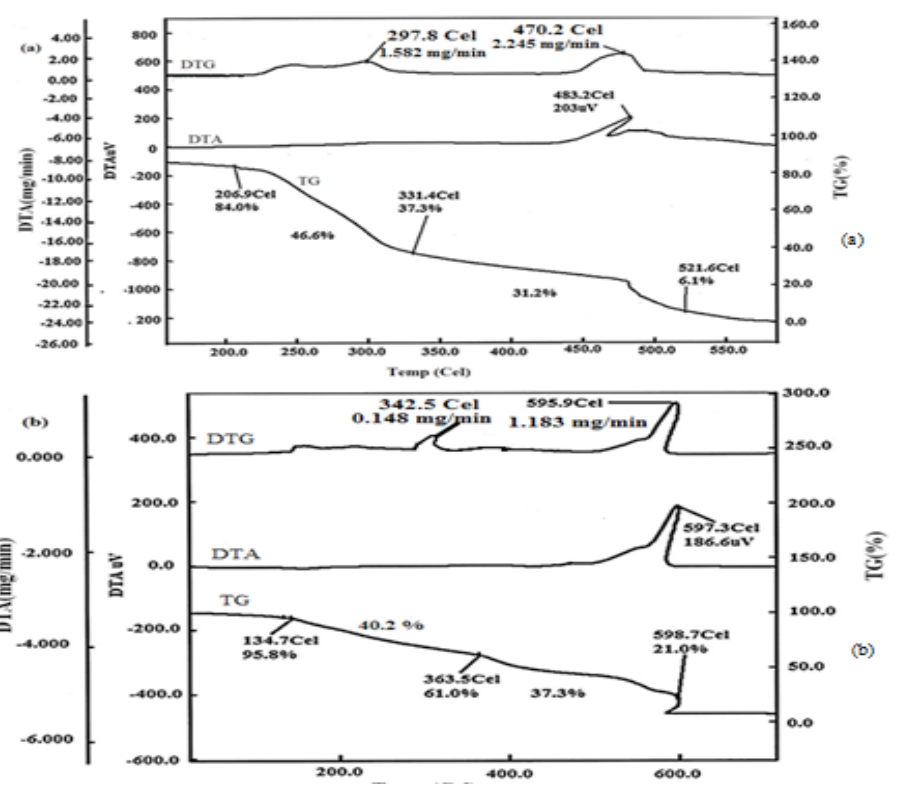

Figure 5: (a) TG/DTA/DTG of GG (b) TG/DTA/DTG of GG-g-PAAM.

DTG of GG showed maximum decomposition at $470.2^{\circ} \mathrm{C}$ with $2.245 \mathrm{mg} /$ min rate of weight loss whereas; in case of GG-g-PAAM maximum decomposition was observed at $595.9^{\circ} \mathrm{C}$ with $1.183 \mathrm{mg} / \mathrm{min}$ rate of weight loss. Therefore, it is clear from the DTG that maximum decomposition was observed at a much higher temperature with a lesser weight loss in GG-g-PAAM compared to GG.

\section{Scanning Electron Microscopy (SEM) analysis}

It was clear from the SEM micrographs of gum ghatti [Figure 6(a)] and that of GG-g-PAAM (G4) [Figure 6(b)] that identifiable morphological change, in the form of transition from flaky structure to porous spongy structure, has taken place due to grafting of PAAM chains onto the polysaccharide. The beads prepared from hydrolysed copolymer were spherical in shape with a rough and dense surface having microscopic cracks on the surface as shown by SEM [Figure 6(c)].
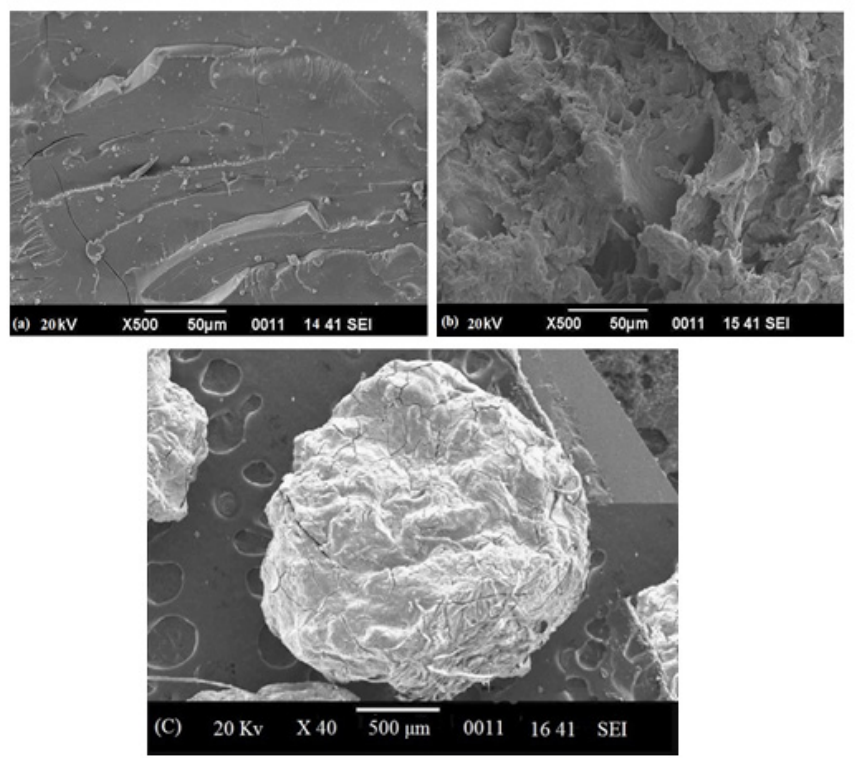

Figure 6: SEM micrograph of (a) gum ghatti (b) GG-g-PAAM (G4) (c) P5 bead.

\section{Estimation and interpretation of intrinsic viscosity}

The intrinsic viscosity was calculated for gum ghatti and for all the batches of GG-g-PAAM, as shown in Table 4.

Intrinsic viscosity is basically the hydrodynamic volume of a molecule in the solvent (water in this case). As shown in Table 4, intrinsic viscosities of all the batches of GG-g-PAAM are higher than that of GG. This is due to the increase in hydrodynamic volume attributed to the grafting of the PAAM chains on the main polymer backbone (GG). The grafted PAAM chains may increase hydrodynamic volume by two ways:

(1) By uncoiling of the polysaccharide chain through steric hindrance to intra-molecular bonding.

(2) By contributing their own hydrodynamic volume.

Table 4: Intrinsic viscosity results of GG-g-PAAM and native GG

\begin{tabular}{cc}
\hline Batch code & Intrinsic viscosity $(\mathrm{dl} / \mathrm{g})$ \\
\hline G1 & 1.72 \\
G2 & 1.95 \\
G3 & 2.52 \\
G4 & 5.47 \\
G5 & 1.80 \\
G6 & 4.12 \\
G7 & 3.43 \\
G8 & 2.16 \\
GG & 1.65 \\
\hline
\end{tabular}

Further, the rise in intrinsic viscosity is because the grafting is in good accord with Mark-Houwink-Sakurada relationship (intrinsic viscosity $\eta=\mathrm{KM}^{\alpha}$, where $\mathrm{K}$ and $\alpha$ are constants, both are related to stiffness of the polymer chains), which features the increase in intrinsic viscosity to the increase in molecular weight $(\mathrm{M})$ due to the grafted PAAM chains. As evident from Figure 7, intrinsic viscosity increases exponentially with percentage grafting.

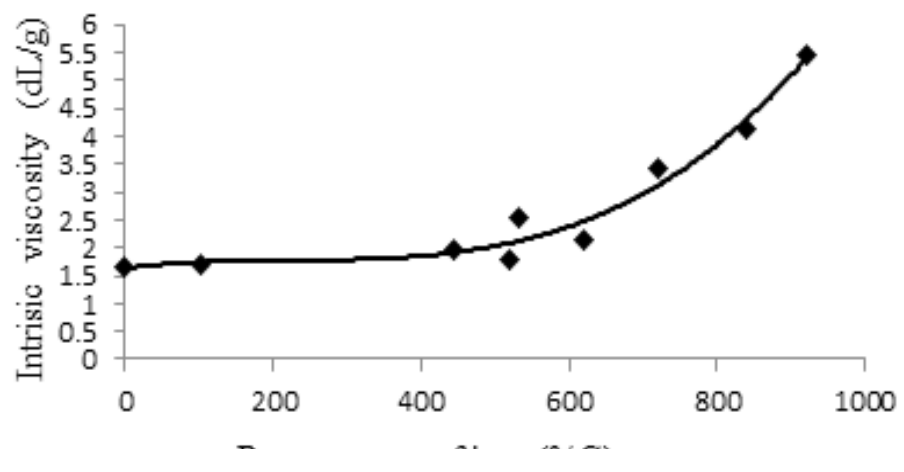

Figure 7: Intrinsic viscosity versus percentage grafting plot for GG-g-PAAM.

Mathematical relationship produced using MLRA for viscosity was given as (Equation 10).

$$
\text { Viscosity }=0.82-0.10 \mathrm{C}+0.058 \mathrm{AC}-0.089 \mathrm{BC}-0.080 \mathrm{ABC}
$$

Where A is amount of CAN (mg), B is irradiation time (min) and C is amount of PAAM (g). From ANOVA study, it was found that the factorial model was significant $(P<0.05)$ having $R^{2}$ value of 0.95 . The adjusted (0.94) and the predicted $(0.85) \mathrm{R}^{2}$ values were in convincingly good 
agreement. Hence it can be understood that the model can be used to follow the design space.

\section{Molecular weight analysis}

The result of gel permeation chromatography (GPC) is shown in Table 5. Most of the batches of grafted gum were eluted at about $7.5-8 \mathrm{~mL}$ of elution volume.

Table 5: Molecular weight analysis data of GG-g-PAAM and native GG

\begin{tabular}{cc}
\hline Batch code & Molecular weight $(\times 106 \mathrm{~g} / \mathrm{mol})$ \\
\hline G1 & 3.2606 \\
G2 & 5.1382 \\
G3 & 4.3490 \\
G4 & 6.0932 \\
G5 & 12.1538 \\
G6 & 8.5782 \\
G7 & 7.5397 \\
G8 & 11.9730 \\
GG & 13.6178 \\
\hline
\end{tabular}

From the results it can be said that the pure gum was having the highest molecular weight of $12.1538 \times 10^{6} \mathrm{~g} / \mathrm{mol}$. However, in case of grafted gum, molecular weight was decreased. In case of optimized batch (G4), molecular weight was found to be $6.0932 \times 10^{6} \mathrm{~g} / \mathrm{mol}$. This extreme reduction in molecular weight was because of the polymer chain distraction during the application of microwave irradiation. Few batches having less \% GE (G5) showed higher molecular weight. This may be due to the presence of pure GG chains in the system. On the other hand, in case of G8, increase in molecular weight was observed though it has a GE of 90.382 $\%$. This was due to the presence of higher amounts of grafted polymer segments in the system. The relation between log transformed value of viscosity and molecular weight was linear $\left(R^{2}=0.97\right)$ which satisfied the Mark-Houwink equation.

\section{Neutralisation equivalent (NE)}

The NE value designates the basicity of polybasic acid. NE is equal to the molecular weight of the acid. During alkaline hydrolysis of the amide groups, molecular weight of the polymer would remain the same (since one - $\mathrm{OH}$ group is incorporated after the exclusion of $\mathrm{NH}_{3}$ ). Therefore, higher the number of carboxyl groups, lower will be the NE. The NE values of pure GG, GG-g-PAAM and hydrolyzed GG-g-PAAM were found to be $1748.63,1740.89$ and 280.32, respectively, (Table 6). This indicates that the hydrolyzed GG-g-PAAM copolymer contains more $\mathrm{COO}_{2}$ groups than pure GG, which are liable for $\mathrm{pH}$ sensitivity.

\section{Swelling studies of GG-g-PAAM in deionized water}

\section{Effect of time on extent of swelling}

Effect of swelling time on Ps was studied at different time intervals (4, $8,12,16,20$ and $24 \mathrm{~h}$ ) in neutral medium at room temperature [Figure

Table 6: Neutralisation equivalent values

\begin{tabular}{cc}
\hline Polymer & NE (g) \\
\hline GG & $1748.63 \pm 0.82$ \\
G4 & $1740.89 \pm 0.68$ \\
Hydrolysed G4 & $280.32 \pm 0.51$ \\
\hline
\end{tabular}

8(a)]. From the figure it was evident that Ps increased with increasing swelling time and attained the maximum value after $16 \mathrm{~h}$, whereas, swelling was found to be arrested on further raise in swelling time. This is due to the fact that the porous structure of the hydrogel got saturated and allowed no more space for the water molecules beyond optimal swelling point.

\section{Effect of temperature on extent of swelling}

The temperature responsive swelling behaviour of GG-g-PAAM was studied at different temperatures $\left(30,40,50,60\right.$ and $\left.70^{\circ} \mathrm{C}\right)$ in neutral medium at pre-optimized time $(16 \mathrm{~h})$ [Figure $8(\mathrm{~b})$ ]. Initially Ps was found to increase with increase in temperature and attained maximum at 60 ${ }^{\circ} \mathrm{C}$ whereas, increase in temperature beyond optimal value resulted in a decreased Ps. At higher temperatures, the elasticity of the polymeric matrix increased so that it can accommodate more water molecules. Also, at higher temperatures, the secondary bonding forces such as hydrogen bonds between water molecules and hydrophilic groups of the hydrogel network plays a prominent role. These hydrogen bonds formed a network like structure and increased the water holding capacity of GG-gPAAM which finally resulted in increased Ps. Nevertheless at too higher temperatures, the polymer matrix gets distorted and the hydrogen bond networks broke down leading to desorption of water molecules and decreased Ps. ${ }^{21}$

\section{Effect of ionic strength of different cations on extent of swelling}

The alteration in the swelling behaviour of GG-g-PAAM with regard to changing ionic strength of different cations in different salt solutions is depicted in Figure 8(c). It was observed from the figure that Ps decreases with increase in ionic strength and even cationic charge. This type of behaviour could be as a result of the reverse osmosis process. Furthermore, value of $\mathrm{C}_{i}^{\mathrm{s}}$ (concentration of the free mobile ions in the medium) increased with increasing ionic strength and reduced the value of $\Pi_{\text {ion }}$ (Osmotic swelling pressure). Therefore, Ps value decreased with increased ionic strength of cations. It was further observed that Ps of GG-g-PAAM decreased with an increase in the charge of metal cations $\left(\mathrm{Na}+>\mathrm{Ba}^{2+}>\right.$
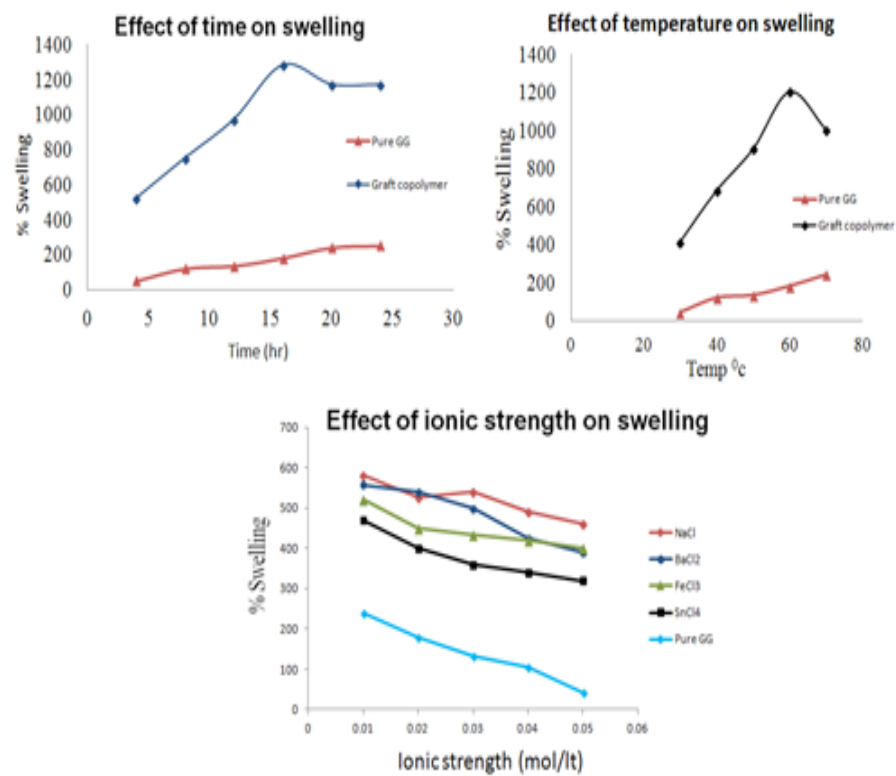

Figure 8: (a) Effect of time on Ps of GG-g-PAAM (b) Effect of temperature on Ps of GG-g-PAAM (c) Effect of ionic strength of various cations on Ps in different salt solutions. 
$\left.\mathrm{Fe}^{3+}>\mathrm{Sn}^{4+}\right)$. Free carboxylate groups of the hydrogel formed complexes with the multivalent cations which reduced the anionic repulsion, and a lesser value of Ps was observed. Therefore, Ps decreased with increased ionic strength of cations and cationic charge.

\section{Measurement of bead size}

The size range of beads was observed to be $980-1215 \mathrm{~mm}$ and was shown in Table 7. $\mathrm{AlCl}_{3}$ acts as a cross-linking agent and as its concentration increased, smaller beads were produced.

This implies that during cross-linking, the hydrogel might have undergone rapid shrinking leading to the formation of smaller and firm matrix with higher cross-link densities. An analogous result has been reported with poly (vinyl alcohol) hydrogels. ${ }^{22}$ Conversely, increase in the amount of Diclofenac sodium increased the bead size as Diclofenac sodium

Table 7: Average bead size and DEE of the beads

\begin{tabular}{ccc}
\hline Beads & Average Size $(\mu \mathrm{m})$ & DEE $(\%)$ \\
\hline P1 & $1215 \pm 2.80$ & $78.18 \pm 0.05$ \\
P2 & $1200 \pm 3.25$ & $77.42 \pm 0.05$ \\
P3 & $996 \pm 3.95$ & $86.17 \pm 0.007$ \\
P4 & $972 \pm 4.38$ & $90.51 \pm 0.01$ \\
P5 & $980 \pm 4.09$ & $89.38 \pm 0.01$ \\
\hline
\end{tabular}

might have occupied the interstitial spaces between polymer segments. This is in accord with the previously published results for chitosan microparticles.

\section{Drug entrapment efficiency}

Table 7 illustrates that Drug entrapment efficiency (DEE) of the beads made with lower concentration of $\mathrm{AlCl}_{3}$ was lowest than those prepared with higher concentration of $\mathrm{AlCl}_{3}$. At lower concentration of $\mathrm{AlCl}_{3}$, the hydrogel matrix might be loose and have larger pores due to inadequate cross-linking, which results in higher seepage of drug into the gelation medium from the polymer matrix during the preparation of beads, and this might have resulted in lower DEE. At higher concentration of $\mathrm{AlCl}_{3}$, the hydrogel matrix is rigid hence seepage of drug from the polymer matrix is low resulting in high DEE. A similar study on DEE of alginate beads has been reported. ${ }^{23}$

\section{Pulsatile swelling behaviour of beads}

The $\mathrm{pH}$ sensitivity of the copolymer was determined by studying pulsatile swelling of drug-free copolymeric beads in the solutions of $\mathrm{pH} 1.2$ and 7.4. Figure 9 depicts that the beads formed from GG did not show noticeable pulsatile swelling behaviour. In contrast, beads made of hydrolysed copolymer swelled and de-swelled sharply with the changing $\mathrm{pH}$ environment. At higher $\mathrm{pH}$ values, the carboxyl functional groups of copolymeric beads undergo ionization and subsequently, the osmotic pressure inside the beads increases resulting in higher swelling. However, the swelling depends upon the density of cross-linking. At low cross-link density, the hydrogel network is loose with a greater hydrodynamic free volume and can absorb more of the solvent resulting in higher swelling.

\section{In vitro drug release}

The release of Diclofenac sodium from pure GG and copolymeric beads in acidic medium was lesser than the analogous beads in alkaline medium (Figure 10). Slower release in acidic medium may be primarily due to the lower solubility of drug in acidic medium (pKa 4.6). Figure 10 further indicates that the drug release from copolymeric beads was significantly lesser than from pure GG beads. This indicates that in addition

\section{Pulsatile swelling behaviour of beads}

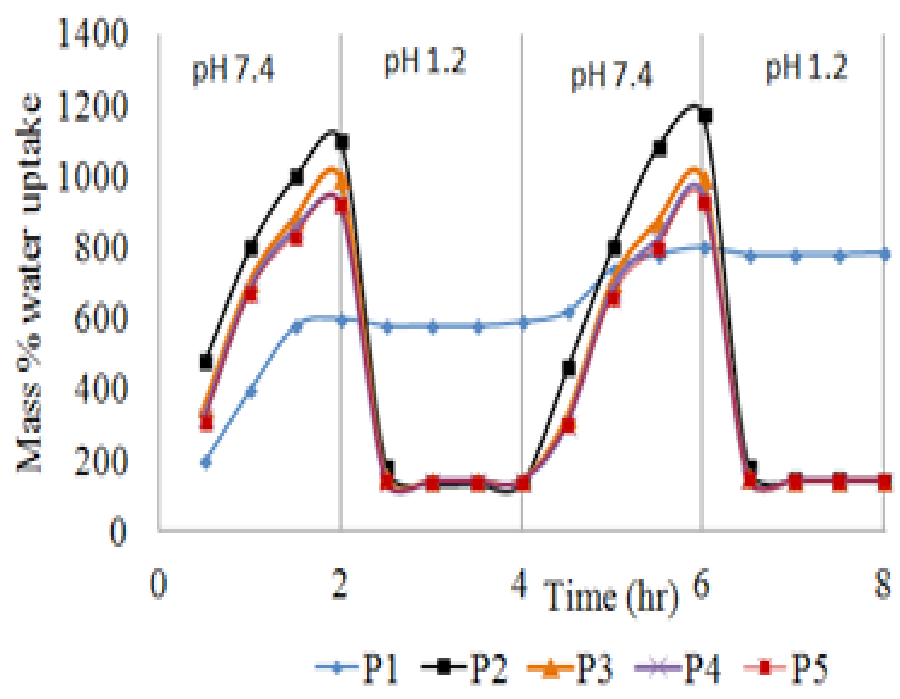

Figure 9: Pulsatile swelling behavior of beads P1 ( $\mathbf{\Delta})$, P2 (घ), P3 ( $\mathbf{\Delta})$, P4 (×) and P5 ( $)$.

to the solubility of drug, swelling behaviour of the beads might have contributed in determining the drug release. Pulsatile swelling study (Figure 9) showed that the copolymeric beads de-swelled rapidly in acidic medium. In other words, the swelling of pure GG beads in acidic medium was greater than that of copolymeric beads and, therefore the drug release was faster from pure GG beads.

The drug release from pure GG beads in alkaline medium was also found to be higher than that from the different batches of copolymeric beads. While pure GG beads released the drug completely in $4 \mathrm{~h}$, a maximum of $90.18 \%$ drug release was observed from the copolymeric beads at the end of $8 \mathrm{~h}$. Although the swelling of copolymeric beads in alkaline medium was higher than that of pure GG beads, slower drug release from the copolymeric beads may be attributed to slower diffusion of drug through

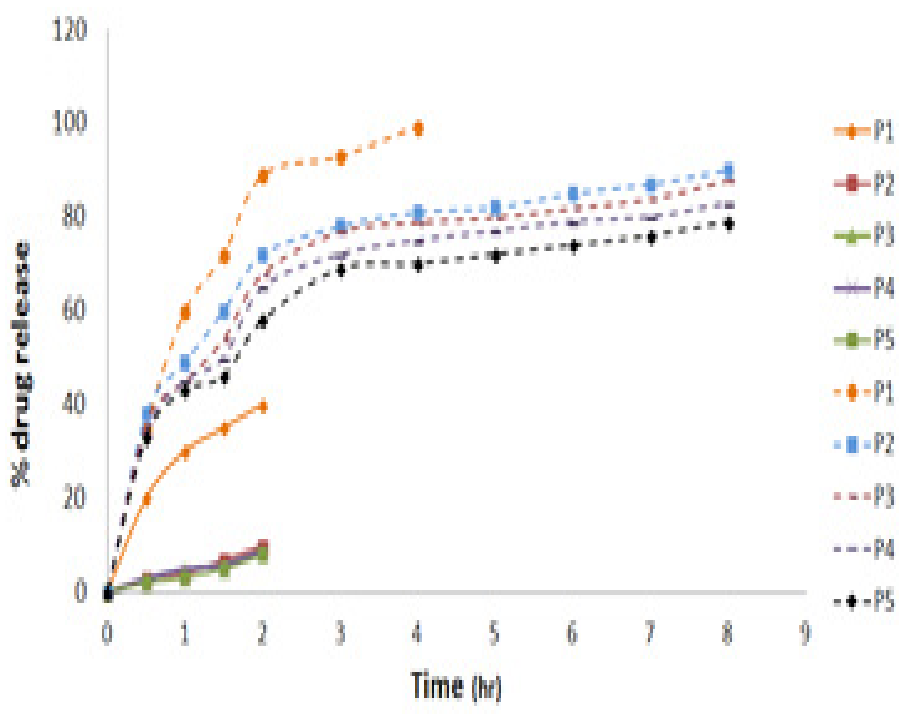

Figure 10: Release of diclofenac sodium from beads in $\mathrm{pH} 1.2$ buffer solution (solid lines) and $\mathrm{pH} 7.4$ buffer solution (dotted lines). 
the rigid hydrogel network. Figure 10 shows that the beads prepared with higher concentration of $\mathrm{AlCl}_{3}$ released the drug more slowly as the increase in the concentration of the gel-forming ions provided high rigidity of the hydrogel network due to the increased cross-link density. The diffusion coefficient ${ }^{24}$ of the drug from various copolymeric beads was calculated using the equation 11 and the results are presented in Table 8 .
Where $\theta$ is the slope of the linear portion of the plot of $\mathrm{M}_{\mathrm{t}} / \mathrm{M}_{\infty}$ versus $\mathrm{t}^{1 / 2}$, $r$ is the radius of the beads and $M_{\infty}$ is the total amount of drug loaded. The diffusion coefficients were determined based on the Fickian diffusion model, and the results of Table 8 showed that the diffusion coefficient of the drug was higher from the beads which were prepared with lower concentration of $\mathrm{AlCl}_{3}$ and as the concentration of $\mathrm{AlCl}_{3}$ increased, diffusion coefficient of the drug decreased considerably $(\mathrm{P}<0.06)$. This

Table 8: Diffusion coefficients (D) and release parameter $(n)$ of the beads in different $\mathrm{pH}$ solutions

\begin{tabular}{cccccccc}
\hline \multirow{2}{*}{ Beads } & \multicolumn{2}{c}{$\mathrm{D}\left(\mathrm{cm}^{2} / \mathrm{s}\right)$} & \multicolumn{3}{c}{$\mathrm{n}$} & \multicolumn{2}{c}{$\mathrm{r}^{*}$} \\
\cline { 2 - 7 } & $\mathrm{pH} \mathrm{1.2}$ & $\mathrm{pH} \mathrm{7.4}$ & $\mathrm{pH} \mathrm{1.2}$ & $\mathrm{pH} \mathrm{7.4}$ & $\mathrm{pH} \mathrm{1.2}$ & $\mathrm{pH} \mathrm{7.4}$ \\
\hline P1 & $6.215 \times 10^{-5}$ & $2.175 \times 10^{-6}$ & 0.678 & 0.628 & 1.192 & 0.985 \\
P2 & $5.318 \times 10^{-6}$ & $7.978 \times 10^{-7}$ & 0.983 & 0.596 & 1.527 & 0.924 \\
P3 & $3.543 \times 10^{-6}$ & $7.287 \times 10^{-7}$ & 1.086 & 0.681 & 0.986 & 0.951 \\
P4 & $2.283 \times 10^{-6}$ & $6.355 \times 10^{-7}$ & 1.315 & 0.733 & 0.954 & 0.883 \\
P5 & $3.179 \times 10^{-6}$ & $7.238 \times 10^{-7}$ & 0.972 & 0.699 & 1.258 & 0.839 \\
\hline
\end{tabular}

${ }^{*}$ Correlation coefficient

$$
\mathrm{D}=\left(\frac{\mathrm{r} \theta}{6 \mathrm{M}_{\infty}}\right)^{2} \pi
$$

result confirms that as the concentration of $\mathrm{AlCl}_{3}$ is increased, more rigid gel structure within the copolymeric beads is formed and this hinders the drug diffusion. Figure 10 also shows the effect of initial drug loading on the release of drug from copolymeric beads. Keeping all the variables constant, increase in initial drug loading increased the drug release. Increase in initial drug load decreases the proportion of polymer per unit weight and this weakens the gel network structure. Moreover, higher drug loading increases the free volume within the network and creates a more tortuous path for water to penetrate through. Consequently, increase in initial drug loading increased the release of drug.

Figure 11 represents the release profiles of Diclofenac sodium from various beads in dissolution medium, the $\mathrm{pH}$ of which was changed at predetermined time intervals. It was evident that at lower $\mathrm{pH}$, the initial drug release was lesser from the copolymeric beads and as $\mathrm{pH}$ increased, the drug release increased and extended over a prolonged period depending upon the formulation variables. To understand the drug release mechanism in the hydrogel network, release data were fitted to an empirical equation $12 .^{25}$

Where $M_{t}$ is the amount of drug released at time $t, M_{\infty}$ is the total amount of drug loaded and $\mathrm{n}$ values are the indication of the type of release mechanism. The calculated $\mathrm{n}$ values along with the correlation coefficients are shown in Table 8.

$$
\frac{M_{t}}{M_{\infty}}=K t^{n}
$$

The values of $\mathrm{n}$ depend upon the cross-link density and increase with increase in cross-link density. The calculated $n$ values suggested that the mechanism of drug release was deviated from the Fickian mode and followed case II transport and anomalous transport, in acidic and alkaline media respectively. P5 bead formulation had demonstrated satisfactory in vitro drug release of 8 and $79 \%$ at the end of $2^{\text {nd }}$ and $8^{\text {th }} \mathrm{h}$ of dissolution study.

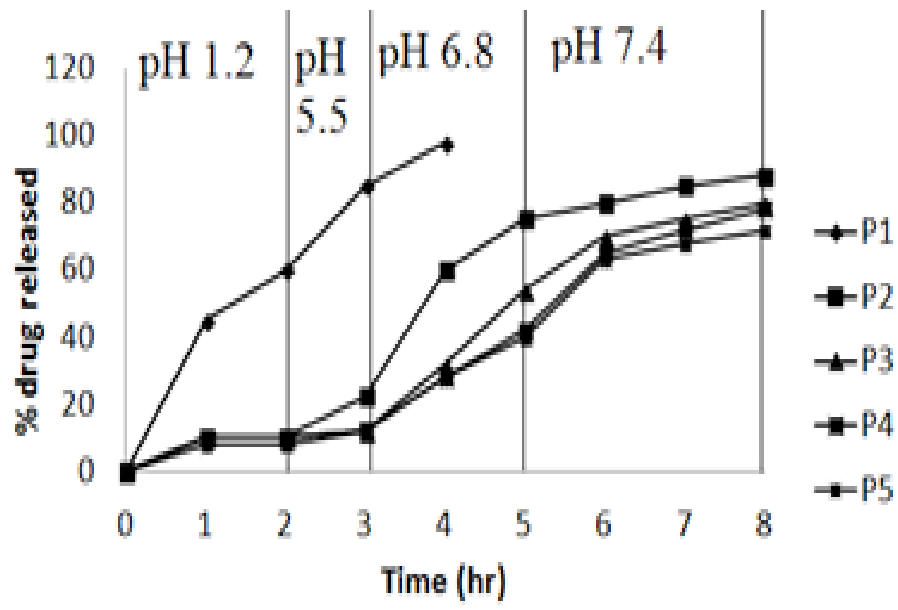

Figure 11: Release of diclofenac sodium from beads during continuous exposure to a changing $\mathrm{pH}$ environment.

\section{Biodegradability study}

Figure 12 shows the fungal growth for different batches of films of GGg-PAAM. It is clearly noticeable in the figures, which show apparent fungi growth for 3, 7, 14 and 21 days in all the Petri dishes. The apparent growth of fungi in mineral salts agar medium (without any added carbon source) proves that the carbon present in the grafted polymer had been utilized by the fungi for its growth. Thus it can be simply concluded that polyacrylamide grafted GG is biodegradable in nature.

\section{Acute oral toxicity study}

The results of acute toxicity studies were shown in Table 9. It is clear that there was no mortality found within the observation period of 14 days after dosing. As per the "Organization of Economic Co-operation and Development (OECD) guideline for the test of chemicals" 425, adopted "17 December 2001" Annexure - 4, considering the need to guard animal welfare; testing in animals is discouraged. As per the globally harmonized system (GHS), if $\mathrm{LD}_{50}$ value is greater than the $2000 \mathrm{mg} / \mathrm{kg}$ dose, then the test product will fall under "Category 5 " and the toxicity 
Table 9: Mortality rate of animals after a single dose of $2000 \mathrm{mg} / \mathrm{kg}$ body weight

\begin{tabular}{cccccc}
\hline Observation time period & \multicolumn{5}{c}{ Mortality } \\
\cline { 2 - 6 } & Animal 1 & Animal 2 & Animal 3 & Animal 4 & Animal 5 \\
\hline 30 min & 0 & 0 & 0 & 0 & 0 \\
4 th & 0 & 0 & 0 & 0 & 0 \\
1st day & 0 & 0 & 0 & 0 & 0 \\
3rd day & 0 & 0 & 0 & 0 & 0 \\
7th day & 0 & 0 & 0 & 0 & 0 \\
14th day & 0 & 0 & 0 & 0 & 0 \\
\hline
\end{tabular}

' 0 ' survival and ' $\mathrm{x}$ ' death

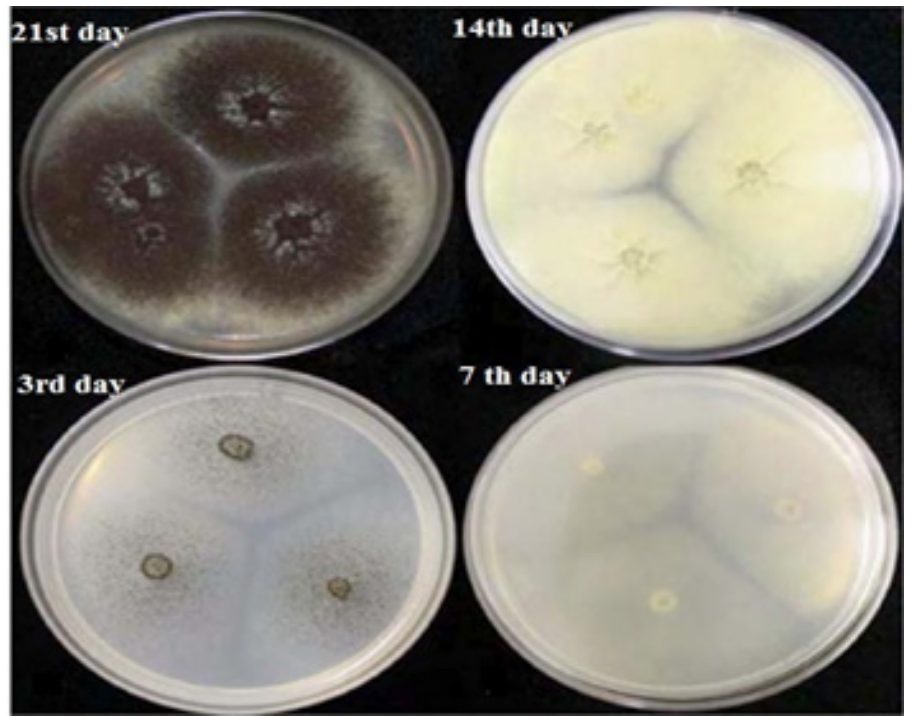

Figure 12: Gradual growth of A. niger in minimal salt agar media by utilizing the grafted gum as carbon source.

rating will be "zero". So, GG-g-PAAM falls under "category 5" with toxicity rating "zero”.

\section{CONCLUSION}

In the present study GG-g-PAAM was synthesized by microwave irradiation using ceric ammonium nitrate as a redox initiator. Optimised GG-g-PAAM copolymer was then hydrolyzed using sodium hydroxide to convert $\mathrm{CONH}_{2}$ groups of PAAM to $\mathrm{COOH}$ groups so as to attain a $\mathrm{pH}$-sensitive copolymer. The release of Diclofenac sodium from the copolymeric beads was considerably less in acidic medium and tended to increase as the $\mathrm{pH}$ of release medium was increased. Major portion of the drug (about 90\%) was found to be released in the $\mathrm{pH}$ range existing in the intestine.

\section{CONFLICT OF INTEREST}

The authors declare that they have no conflict of interest

\section{ACKNOWLEDGEMENT}

The authors acknowledge JSS Mahavidyapeetha, Mysuru and JSS University, Mysuru, India, for their valuable support during this research.
The authors wish to thank CFTRI, Mysuru for providing facilities for SEM and DSC studies.

\section{ABBREVIATIONS USED}

DS: Diclofenac sodium; FT-IR: Fourier transform infrared; SEM: Scanning electron microscopy; DSC: Differential scanning calorimetry; GG: ghatti gum; NSAID: Non-steroidal anti-inflammatory drug; GIT: Gastrointestinal tract; PAAM: Polyacrylamide; CAN: Ceric ammonium nitrate; GE: Grafting efficiency; MLRA: Multiple linear regression analysis; FDT: Final decomposition temperature; IDT: Initial decomposition temperature; GPC: Gel permeation chromatography; DEE: Drug entrapment efficiency.

\section{REFERENCES}

1. Bechgaard $H$, Hegermann NG. Controlled release multipleunits and single unit doses: A literature review. Drug Dev Ind Pharm. 1978;4(1):53-67.

2. Ghebre-Sellassie I. Multiple oral drug delivery. 1994; Marcel Dekker: New York.

3. Galeone M, Nizzola, I, Cacioli D, Mosie G. In vitro demonstration of delivery mechanism from sustained release pellets. Curr Ther Res. 1981;29(2):217-34.

4. Davis SS, Hardy JG, Taylor MJ, Whalley DR, Wilson CG. Comparative study of the gastrointestinal transit of a pellet and tablet formulation. Int J Pharm. 1984; 21(2):167-177.

5. Fowler PD. Aspirin, paracetamol and non-steroidal anti-inflammatory drugs: A 
comparative review of side effects. Med Toxic. 1987;2(5):338-66.

6. Cook PM. Side effects of non-steroidal anti-inflammatory drugs. Med J Austral. 1988;148:248-51.

7. Desai KG, Liu C, Park HJJ. Characteristics of vitamin C encapsulated tripolyphosphate-chitosan microspheres as affected by chitosan molecular weight. Microencapsul. 2006;23(1):79-90.

8. Qiu Y, Park K. Environment sensitive hydrogels for drug delivery. Adv Drug Deliv Rev. 2001;53(3):321-39.

9. Kaur $\mathrm{H}$, Chatterji RR. Interpenetrating hydrogel networks- swelling and mechanical properties of the gelatin-polyacrylamide interpenetrating networks. Macromolecules. 1990;23(22):4868-71.

10. Anand S, Deshmukha C, Mallikarjuna S, Aravind M, Badigerc KS, Muralikrishna K. Gum ghatti: A promising polysaccharide for pharmaceutical applications. Carbohydr Polym. 2012;87(2):980-6.

11. Athawale VD, Lele V. Graft co-polymerization onto starch. II: Grafting of acrylic acid and preparation of its hydrogels. Carbohydr Polym. 1998;35(1-2):21-7.

12. Kaity S, Isaac PJ, Kumar M, Bose A, Wong TW, Ghosh A. Microwave assisted synthesis of acrylamide grafted locust bean gum and its application in drug delivery. Carbohydr Polym. 2013;98(1):1083-94

13. Raghavendra $V$, Kulkarni B. Enteric delivery of ketoprofen through functionally modified poly (acrylamide-grafted-xanthan)-based $\mathrm{pH}$-sensitive hydrogel beads: Preparation, in vitro and in vivo evaluation. J Drug Target. 2008;16(2):167-77.

14. Robert MS, Francis XW. Spectrometric identification of organic compounds. 2011; Wiley: New Delhi.

15. Collins EA, Bares J, Billmeyer FW. Experiments in polymer science. 1973; John Wiley \& Sons: New York.

16. Xu FJ, Kang ET, Koon-Gee N. pH- and temperature-responsive hydrogels from crosslinked triblock copolymers prepared via consecutive atom transfer radical polymerizations. Biomater. 2006;27(14):2787-97.

17. Tripathy T, Singh RP. High performance flocculating agent based on partially hy- drolysed sodium alginate-g-polyacrylamide. Eur Polym J. 2000;36(7):1471-76.

18. Tian D, Xie HQ. Synthesis and Flocculation Characteristics of Konjac Glucomannan-g-Polyacrylamide. Polym Bull. 2008;61(3):277-85.

19. Kaith BS, Jindal R, Mittal H, Kumar K. Synthesis, characterization, and swelling behavior evaluation of hydrogels based on gum ghatti and acrylamide for selective absorption of saline from different petroleum fraction-saline emulsions. J Appl Polym Sci. 2012;124(3):2037-47.

20. Kaith BS, Jindal R, Maiti M, Jana AK. Development of corn starch based green composites reinforced with Saccharum spontaneum $L$ fiber and graft copolymers-Evaluation of thermal, physico-chemical and mechanical properties. Bioresour Technol. 2010;101(17);6843-51.

21. Fu-Jian X, En-Tang K, Koon-Gee N. pH- and temperature-responsive hydrogels from crosslinked triblock copolymers prepared via consecutive atom transfer radical polymerizations. Biomater. 2007;27(14):2787-97.

22. Korsmeyer RC, Peppas NA. Effect of the morphology of hydrophilic polymeric matrices on the diffusion and release of water-soluble drugs. J Membr Sci. 1981;9(3):211-27.

23. Bhopatkar D, Anal AK, Stevens WF. Ionotropic alginate beads for controlled intestinal protein delivery: Effect of chitosan and barium counter-ions on entrapment and release. J Microencapsul. 2005;22(1):91-100.

24. Agnihotri SA, Aminabhavi TM. Controlled release of clozapine through chitosan microparticles prepared by novel method. J Control Rel. 2004;96(2):245-59.

25. Ritger PL, Peppas NA. A simple equation for description of solute release. II. Fickian and anomalous release from swellable devices. J Control Rel. $1987 ; 5(1): 37-42$.

Article History: Submission Date : 17-08-2017 ; Revised Date : 29-08-2017; Acceptance Date : 12-09-2017.

Cite this article: Moin A, Gowda DV. Enteric Delivery of Diclofenac Sodium through Functionally Modified Poly (acrylamide-grafted-Ghatti gum)-based pHsensitive Hydrogel Beads: Development, Formulation and Evaluation J Young Pharm. 2017;9(4):525-36. 